\title{
Theoretical study of the effect of substituent and backbone conformation on the electronic properties of symmetrically substituted poly(di- $n$-alkylsilanes)
}

\author{
Raúl Crespo, Mari Carmen Piqueras, and Francisco Tomás \\ Departament de Química Física, Universitat de València, Dr. Moliner 50, E-46100 Burjassot, \\ Valencia, Spain
}

(Received 3 September 1993; accepted 20 January 1994)

\begin{abstract}
We present the results of $a b$ initio $3-21 \mathrm{G}^{*}$ geometry optimizations and valence effective Hamiltonian (VEH) band structure calculations aimed at determining the evolution of the geometric and electronic (ionization potential, electron affinities, and band gaps) properties of all-trans poly(dimethylsilane), poly(diethylsilane), poly(di- $n$-propylsilane), and poly(di- $n$-butylsilane) when increasing the size of the alkyl group. In the latter polymer, we have also studied the $7 / 3$ conformation, in order to analyze the effect of the backbone conformation on the geometric and electronic structure. The VEH ionization potentials of all-trans poly(di- $n$-alkylsilanes) are almost equal, and as experimental photoemission data show, only slight differences are appreciated. The band gap decreases in going from poly(di- $n$-butylsilane) to poly(di- $n$-propylsilane) and to poly(diethylsilane), and increases when passing to poly(dimethylsilane), which coincides with experimental evidences on poly(di-n-alkylsilanes). The change from all-trans to $7 / 3$ conformation of poly(di-n-butylsilane) implies an increase of both, ionization potential and band gap, in perfect agreement with experimental photoemission and absorption data. The applicability of VEH to deal with poly(di- $n$-alkylsilanes) is discussed.
\end{abstract}

\section{INTRODUCTION}

Polymer chemistry and technology form one of the major areas of materials science. Most of the polymers included in this field are conjugated organic materials mainly composed of carbon atoms linked together or separated by heteroatoms such as oxygen or nitrogen. ${ }^{1}$ In this context, organopolysilanes are linear polymers whose backbone is entirely made up of silicon atoms, ${ }^{2}$ which structurally, are more closely related to homoatomic organic polymers such as polyolefines. They can be obtained as homopolymers or copolymers, soluble in common organic solvents, that present two pendant groups which are typically organic units as alkyl and aryl groups. ${ }^{2.3}$ The properties of these materials depend greatly on the nature of the substituent groups. They show considerable promise as ultraviolet acting photoresist for microelectronics, ${ }^{4}$ and as photoconductors in electrophotography. ${ }^{5}$ They are active as free radical photoinitiators for polymerization reactions, ${ }^{6}$ and show marked nonlinear optical properties. ${ }^{7}$ Furthermore, organopolysilanes differ from all other high polymers in that they exhibit sigmadelocalization, that leads to special physical properties as strong electronic absorption, photoconductivity, and photosensitivity, that determine many of their technological applications. ${ }^{2,3}$

Experimental works have demonstrated that the wavelength of maximum absorption, $\lambda_{\max }$, increases with increasing the length of the silicon backbone, ${ }^{8}$ as well as with increasing the size of substituents. ${ }^{9}$ Furthermore, these polymers present thermochromic behavior in both, solution and solid state. In solution, some organopolysilanes show a reversible thermochromism, with $\lambda_{\max }$ usually moving to larger wavelength as the temperature decreases, that is attributed to an increase of the portion of trans conformations at lower temperatures. ${ }^{10}$ In poly(di-n-alkylsilanes) a strong and discontinuous wavelength shift is observed experimentally over a relatively narrow temperature range. ${ }^{11}$ These results suggest that two conformational isomers must coexist in solution, and has been explained as a coil-to-rod transformation or to crystallization of the side chains, either of which would lead to a larger proportion of trans-junctions in the lowtemperature form. ${ }^{11}$

In solid state, the most studied organopolysilane is poly (di- $n$-hexylsilane) (PDHS). Interest in this polymer arose from the discovery that it presents a striking change in ultraviolet absorption spectrum at $42^{\circ} \mathrm{C}$. Above this temperature the $\lambda_{\max }$ of PDHS is $317 \mathrm{~nm}$ and below this temperature a new phase that presents a $\lambda_{\max }$ of $374 \mathrm{~nm}$ grows. ${ }^{12} \mathrm{X}$-ray diffraction, electron diffraction, RMN, and Raman spectroscopy studies have shown that the low-temperature form is highly crystalline with an all-trans arrangement of the silicon backbone, while at high temperatures a disordered phase is observed. ${ }^{13}$ For poly(di- $n$-heptylsilane) (PDHepS) and poly(di-n-octylsilane) (PDOS) the same behavior as PDHS is observed. $^{14}$ X-ray studies show that solid poly(di-npropylsilane) (PDPrS), ${ }^{15}$ poly(diethylsilane) (PDES), ${ }^{15}$ and poly(dimethylsilane) (PDMS) ${ }^{16}$ also present an all-trans conformation. However, the intermediate polymers, poly(di- $n$-butylsilane) (PDBS) ${ }^{17}$ and poly(di- $n$-pentylsilane) (PDPS) ${ }^{17,18}$ are not thermochromic. This feature has been attributed to the fact that these polymers present a structure which is different to that observed in the other poly(di-nalkylsilanes). In fact, the backbone of PDBS and PDPS adopts a helical $7 / 3$ conformation, ${ }^{17,18}$ which implies a twist of approximately $30^{\circ}$ of the dihedral angle on the silicon chain.

Recently, the effect of increasing the pressure on the 
ultraviolet absorption spectrum of poly(di- $n$-alkylsilanes) has been investigated. These studies show that under moderate pressure PDHS films are transformed into the all-trans crystalline form having the $\lambda_{\max }$ at $374 \mathrm{~nm} .{ }^{19,20}$ At atmospheric pressure PDBS ${ }^{17}$ and PDPS ${ }^{17.18}$ display a $\lambda_{\max }$ of $315 \mathrm{~nm}$. With increasing pressure the intensity of the $315 \mathrm{~nm}$ band decreases and a new absorption band develops at $350 \mathrm{~nm}$ for PDBS and $360 \mathrm{~nm}$ for PDPS. ${ }^{21}$ X-ray diffraction ${ }^{19}$ and Raman $^{21}$ studies have determined that this piezochromism is due to a conformational change from a 7/3 helical conformation to an all-trans conformation.

A variety of semiempirical and $a b$ initio methods ${ }^{22-27}$ have been used to investigate the electronic structure of organopolysilanes. These theoretical studies have consisted primarely of unsubstituted polysilane chain, i.e., two hydrogen atoms attached to each silicon atom. In this paper we extend our investigations on polysilanes ${ }^{28-31}$ to the study of poly(di-n-alkylsilanes). We present a detailed investigation of the geometrical and electronic structure of PDMS, PDES, PDPrS, and PDBS. In a first step we analyze the effect of increasing the size of the alkyl chain in the geometrical parameters and electronic structure of the all-trans poly(di- $n$ alkylsilanes), in order to study the bathochromic shift of the absorption maximum. In a second part we turn to the silicon backbone conformation, as is considered to originate the thermochromic and piezochromic behavior, and analyze the changes in both geometrical and electronic structure of PDBS when passing from an all-trans to a $7 / 3$ conformation.

The geometrical parameters of the unit cell of the polymers have been obtained from related oligomers employing $a b$ initio methods as implemented in GAUSSIAN-92. ${ }^{32}$ The electronic structure of the polymers has been calculated using the valence effective Hamiltonian (VEH) theoretical method. ${ }^{33}$ This methodology has been extensively and successfully applied in the field of conducting polymers ${ }^{34,35}$ and has been shown to provide accurate estimates of essential electronic properties, including the energies of the lowest optical transitions. The good agreement between theoretical VEH and experimental results, obtained previously in the study of methylation ${ }^{29}$ and phenylation ${ }^{31}$ of polysilane, has led us to apply the VEH technique to the study of poly(di- $n$ alkylsilanes).

\section{METHODOLOGY}

The electronic band-structure calculations presented in this paper have been performed using the valence effective Hamiltonian (VEH) technique. This method was first developed for molecular calculations by Nicolas and Durand ${ }^{36}$ and extended to polymeric systems by André et al. ${ }^{37}$ In this method, the usual one-electron Fock operator is simulated by a valence effective Fock operator, $F_{\text {eff }}$. This operator is taken as the sum of the kinetic energy, and a summation of atomic potentials of the atoms, within their specific chemical environment, and is written in the polymer case as

$$
F_{\text {eff }}=-\frac{\Delta}{2}+\sum_{g} \sum_{A} V_{A}^{g},
$$

$$
V_{A}=\sum_{l, m, t, u} C_{t u, l m}^{A}\left|\chi_{t l m}^{A}\right\rangle\left\langle\chi_{u l m}^{A}\right|
$$

with the summation over $g$ and $A$ running, respectively, over the polymer unit cells and the atoms present in one cell, and the sum over $l$ and $m$ define the angular dependence of $V_{A}$. The $C_{t u, l m}$ and $\chi_{t l m}$ terms correspond to numerical coefficients and normalized Gaussian functions, respectively.

The atomic potentials are optimized on model molecules, in order to minimize the difference between $F_{\text {eff }}$ and the exact Fock operator. For each molecule the effective Fock operator is constructed with the occupied valence orbitals determined from a minimal basis set, whereas the corresponding energies are obtained from double-zeta basis set calculations. This allows us to obtain one-electron energy levels of $a b$ initio double-zeta quality when we solve the set of secular equations using a minimal basis set. One interesting feature of this methodology is that the highest occupied levels are especially well reproduced. Furthermore as no information pertaining to the excited states is included in the atomic potentials, the virtual levels do not suffer from the usual Hartree-Fock deficiencies. In the Hartree-Fock model the virtual levels are calculated maintaining the $N$ electrons in the occupied levels, i.e., calculating a system with $N+1$ electrons instead of a system with $N$ electrons. It must be stressed that the VEH method is completely nonempirical and not basis set dependent. Since it further avoids the iterative cycles inherent to all SCF calculations, as only valence electrons are explicitly considered and only one-electron integrals need to be evaluated, the method is very fast and economic in computing cost. This allows us to carry out calculations on large-size systems. ${ }^{38}$ Furthermore, the longrange coulombic interactions problem, that exists in ab initio calculation $\mathrm{s}^{39}$ is not present in VEH calculations. ${ }^{40}$

It should be stressed that in order to achieve for an universal parameterization, the determination of the atomic potentials was carried out using different model molecules based on alkyl derivatives of trisilane (trisilane, 2,2'-dimethyltrisilane, 2,2'-diethyltrisilane,...). The parameterization has been successfully applied to different organopolysilanes, ${ }^{28-31}$ providing accurate predictions of essential electronic properties. The good agreement between VEH and experimental ionization potentials and energies of the lowest optical transition demonstrates that VEH provides accurate description of both occupied and unoccupied valence electronic levels. This is an expected feature since the effective Fock operator is constructed to yield $a b$ initio double zeta results only for the occupied levels, and no information pertaining to the unoccupied levels is included in the atomic potentials. ${ }^{40}$ This is a clear indication that part of the electron-hole correlation is included into the virtual oneelectron levels by this methodology. ${ }^{41}$

\section{RESULTS}

\section{A. Geometry optimizations}

As VEH band structure calculations need as input unit cell geometries we have used ab initio Hartree-Fock method to obtain reliable geometrical parameters to build up the 


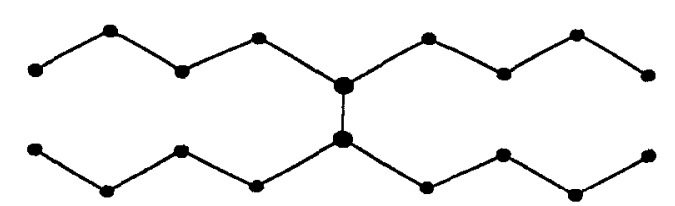

(a)

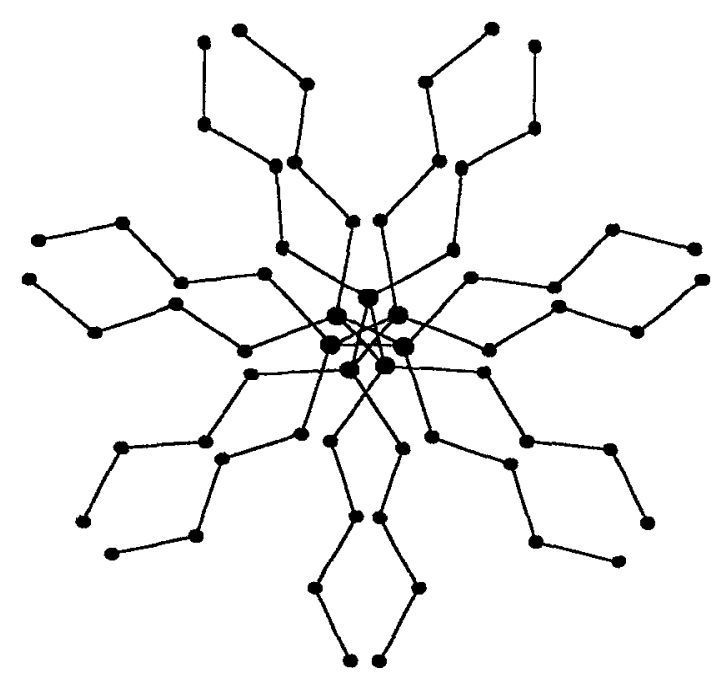

(b)

FIG. 1. Projection of the all-trans (a) and $7 / 3$ (b) helical conformations of poly(di-n-butylsilane) onto a plane normal to the helical axis. Larger dots represent silicon atoms, smaller dots represent carbon atoms.

polymer unit cell. We have followed the same methodological approach successfully applied in previous instances for methyl substituted polysilanes ${ }^{29}$ and poly(phenylsilane), ${ }^{31}$ for which the calculated electronic properties were in excellent agreement with available experimental data. Thus, the geometries of the closely related molecules $1,1^{\prime}, 2,2^{\prime}, 3,3^{\prime}$-hexamethyltrisilane, $1,1^{\prime}, 2,2^{\prime}, 3,3^{\prime}$-hexaethyltrisilane, $1,1^{\prime}, 2,2^{\prime}, 3,3^{\prime}$-hexa- $n$-propyltrisilane, and $1,1^{\prime}, 2,2^{\prime}, 3,3^{\prime}$-hexa- $n$-butyltrisilane have been fully optimized subjected to $C_{2 v}$ symmetry constraint. We have chosen these molecules because geometric optimizations previously reported for $n$-oligosilanes have shown that, the bond lengths and bond angles obtained for trisilane are almost equal to those obtained for longer $n$-oligosilanes. ${ }^{42}$

In all cases the optimizations have been carried out assuming a dihedral angle $\angle \mathrm{C}_{2} \mathrm{C}_{1} \mathrm{SiC}_{1}^{\prime}$ of $180^{\circ}$ except $1,1^{\prime}, 2,2^{\prime}, 3,3^{\prime}$-hexa- $n$-butyl-trisilane for which also has been optimized the molecule with a $\angle \mathrm{C}_{2} \mathrm{C}_{1} \mathrm{SiC}_{1}^{\prime}$ of $0^{\circ}$. These two dihedral angles reproduce, respectively, the dispositions of the alkyl chains in the all-trans and $7 / 3$ conformations of the polymers. The different dispositions adopted by the alkyl chains in PDBS can be observed in Fig. 1, in which a schematic representation of the silicon backbone and alkyl chains dispositions adopted to reproduce the all-trans and 7/3 conformations of PDBS have been displayed. In order to repro-
TABLE I. Geometrical parameters used to build up the unit cells of poly(di$n$-alkylsilanes). Distances are in $\AA$ and angles in degrees.

\begin{tabular}{|c|c|c|c|c|c|c|}
\hline $\begin{array}{c}\text { Polymer } \\
\text { conformation }\end{array}$ & $\begin{array}{l}\mathrm{PS}^{\mathrm{a}} \\
2 / 1\end{array}$ & $\begin{array}{l}\text { PDMS } \\
2 / 1\end{array}$ & $\begin{array}{c}\text { PDES } \\
2 / 1\end{array}$ & $\begin{array}{l}\text { PDPrS } \\
2 / 1\end{array}$ & $\begin{array}{c}\text { PDBS } \\
2 / 1\end{array}$ & $\begin{array}{c}\text { PDBS } \\
7 / 3\end{array}$ \\
\hline \multicolumn{7}{|l|}{ Bond lengths } \\
\hline $\mathrm{Si}-\mathrm{Si}$ & 2.3456 & 2.3522 & 2.3876 & 2.3871 & 2.3876 & 2.3594 \\
\hline $\mathrm{Si}-\mathrm{H}$ & 1.4805 & & & & & \\
\hline $\mathrm{Si}-\mathrm{C}_{1}$ & & 1.9045 & 1.9315 & 1.9322 & 1.9321 & 1.9326 \\
\hline $\mathrm{C}_{1}-\mathrm{C}_{2}$ & & & 1.5442 & 1.5426 & 1.5421 & 1.5496 \\
\hline$C_{2}-C_{3}$ & & & & 1.5436 & 1.5434 & 1.5419 \\
\hline $\mathrm{C}_{3}-\mathrm{C}_{4}$ & & & & & 1.5412 & 1.5412 \\
\hline $\mathrm{C}_{1}-\mathrm{H}_{1}$ & & 1.0877 & 1.0898 & 1.0907 & 1.0907 & 1.0896 \\
\hline $\mathrm{C}_{2}-\mathrm{H}_{2}$ & & & 1.0858 & 1.0808 & 1.0814 & 1.0840 \\
\hline $\mathrm{C}_{3}-\mathrm{H}_{3}$ & & & & 1.0849 & 1.0857 & 1.0857 \\
\hline $\mathrm{C}_{4}-\mathrm{H}_{4}$ & & & & & 1.0851 & 1.0850 \\
\hline $\mathrm{C}_{T}-\mathrm{H}_{T}$ & & 1.0876 & 1.0802 & 1.0851 & 1.0845 & 1.0845 \\
\hline \multicolumn{7}{|l|}{ Bond angles ${ }^{b}$} \\
\hline SiSiSi & 110.91 & 112.93 & 111.57 & 111.30 & 112.18 & 111.20 \\
\hline HSiSi & 109.49 & & & & & \\
\hline $\mathrm{C}_{1} \mathrm{SiSi}$ & & 108.72 & 112.79 & 112.86 & 112.58 & 106.46 \\
\hline $\mathrm{C}_{2} \mathrm{C}_{1} \mathrm{Si}$ & & & 126.30 & 126.68 & 126.50 & 120.40 \\
\hline $\mathrm{C}_{3} \mathrm{C}_{2} \mathrm{C}_{1}$ & & & & 110.79 & 111.37 & 111.61 \\
\hline $\mathrm{C}_{4} \mathrm{C}_{3} \mathrm{C}_{2}$ & & & & & 111.96 & 112.05 \\
\hline
\end{tabular}

${ }^{2}$ Values from Ref. 29.

${ }^{\circ} \angle \mathrm{HCC}$ bond angles are all about $110^{\circ}$ and are not included in the table.

duce the environment of $\mathrm{SiR}_{2}$ units in the polymer, the geometrical parameters used to build up the unit cell of each polymer in the all-trans $\left(\angle \mathrm{SiSiSiSi}=180^{\circ}\right)$ and $7 / 3$ conformation, have been taken from those related to the central silicon atom of the corresponding molecule. The geometrical parameters used to reproduce the backbone of 7/3 PDBS have been obtained maintaining the $\mathrm{Si}-\mathrm{Si}$ bond length and $\angle \mathrm{SiSiSi}$ bond angle calculated for $1,1^{\prime}, 2,2^{\prime}, 3,3^{\prime}$ hexa- $n$-butyltrisilane with $\angle \mathrm{C}_{2} \mathrm{C}_{1} \mathrm{SiC}_{1}^{\prime}=0^{\circ}$, and adopting the $\angle$ SiSiSiSi dihedral angle $\left(148.71^{\circ}\right)$ that yields the $7 / 3$ conformation.

All the geometry optimizations have been performed using the $3-21 \mathrm{G}^{*}$ basis set as implemented in the GAUSSIAN-92 package. ${ }^{32}$ This basis set adds a complete set of six supplementary $d$ Gaussians on silicon atoms, which has been shown to yield equilibrium geometries in excellent agreement with experimental structures for molecules containing second-row elements. ${ }^{42,43}$

Table I collects the geometrical parameters used to build up the unit cells employed in the electronic band structure calculations. As can be seen, the change of hydrogen atoms of polysilane by methyl groups to yicld PDMS, only produces a small increase in the $\mathrm{Si}-\mathrm{Si}$ bond length passing from $2.3456 \AA$ in polysilane to $2.3522 \AA$ in PDMS. However the changes observed in the $\angle \mathrm{SiSiSi}$ bond angle are more accentuated $\left(110.91^{\circ}-112.93^{\circ}\right)$, because of the steric hindrance between methyl groups in 1 and 3 positions.

The substitution of methyl groups by ethyl groups shows important changes in the geometrical parameters. The $\mathrm{Si}-\mathrm{Si}$ bond length increases from $2.3522 \AA$ in PDMS to $2.3876 \AA$ in PDES and the $\mathrm{Si}-\mathrm{C}_{1}$ bond length increases from 1.9045 to $1.9315 \AA$, when passing from PDMS to PDES. The $\mathrm{C}_{1} \mathrm{SiSi}$ bond angle shows an important increase, from $108.72^{\circ}$ to $112.79^{\circ}$, in going from PDMS to PDES, and consequently 


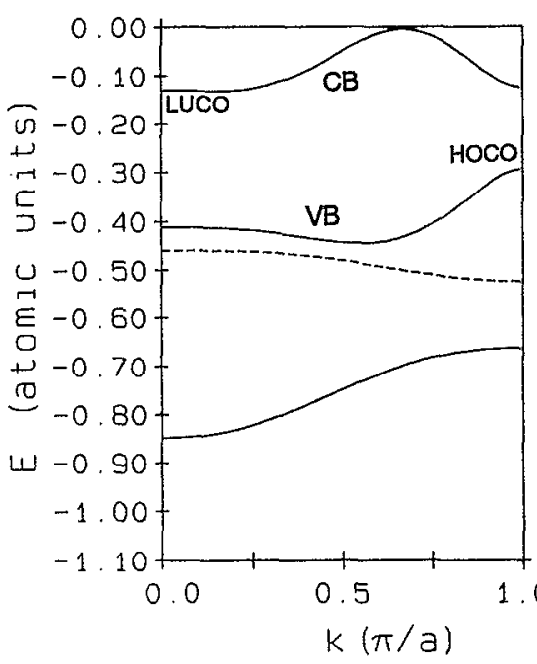

(a)

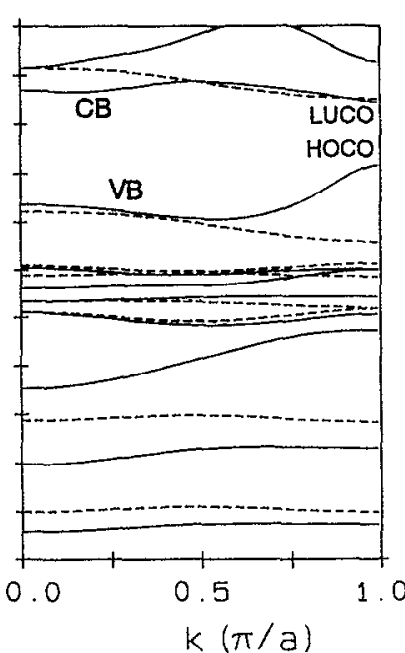

(b)

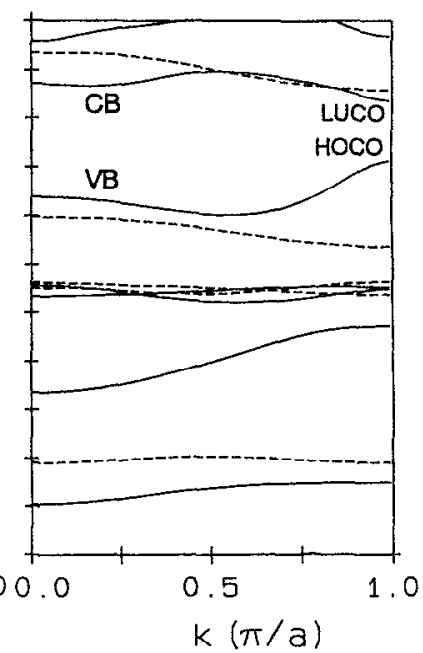

(c)

FIG. 2. VEH band stuuctures calculated for all-truns polysilane (a), poly(diethylsilane) (b), and poly(dimethylsilane) (c). Energies are in atomic units. Solid and dashed lines denote symmetric and antisymmetric bands respect to the backbone plane, respectively. Unoccupied bands with energies larger than 0.0 atomic units are not shown. VB denotes valence band and CB denotes conduction band. HOCO and LUCO denote the highest occupied and lowest unoccupied crystal orbital, respectively.

the $\angle$ SiSiSi bond angle slightly decreases from $112.93^{\circ}$ to $111.57^{\circ}$. These important changes are due to the strong interaction between the hydrogen atoms of the terminal carbon of the ethyl group that are linked to consecutive silicon atoms that appear in PDES. Moreover, this interaction produces that the $\mathrm{C}_{2} \mathrm{C}_{1} \mathrm{Si}$ bond angle of PDES displays an important deviation from a typical tetrahedral angle $\left(109.47^{\circ}\right)$. This very larger value $\left(126.30^{\circ}\right)$ increases the distance between the hydrogen atoms and decreases this interaction.

The geometrical parameters calculated for PDPrS and PDBS are very similar to that obtained for PDES, being the differences in the bond lengths and bond angles less than $0.001 \AA$ and $0.5^{\circ}$, respectively. This is an expected result since the incorporation of a new methylene group in the alkyl chain, in going from PDES to PDPrS and to PDBS, does not introduce any strong additional interaction between hydrogens of adjacent silicon atoms.

The geometrical parameters obtained for PDBS in the $7 / 3$ conformation show that the bond lengths are very similar to those calculated for the all-trans, except for the siliconsilicon bond length $(2.3594$ and $2.3876 \AA$ in $7 / 3$ and alltrans, respectively). The $\mathrm{Si}-\mathrm{Si}$ value calculated for the $7 / 3$ conformation is almost equal to that obtained for all-trans PDMS (2.3522 $\AA$ ). This is due to, as in all-trans PDMS, in $7 / 3$ PDBS the interaction between the alkyl chains linked to consecutive silicon atoms, that takes place in the all-trans conformation of the other poly(di-n-alkylsilanes), is not present. The bond angles of both $7 / 3$ and all-trans PDBS show that the larger differences are found in $\angle \mathrm{C}_{1} \mathrm{SiSi}$ and $\angle \mathrm{C}_{2} \mathrm{C}_{1} \mathrm{Si}$ angles. These angles decrease about $6.1^{\circ}$ in going from all-trans to $7 / 3$ PDBS because in the $7 / 3$ conformation the interaction between the alkyl chains is due to the two chains linked to the same silicon atom, but in the all-trans conformation this interaction takes place between alkyl chains linked to consecutive silicon atoms (see Fig. 1). Therefore, the decrease of the $\angle \mathrm{C}_{1} \mathrm{SiSi}$ bond angle in going from all-trans to 7/3 PDBS, produces an increase of the distance between the alkyl chains and a decrease of the interactions between them. The $\angle \mathrm{C}_{2} \mathrm{C}_{1} \mathrm{Si}$ angle in the $7 / 3$ conformation $\left(120.40^{\circ}\right)$ is also larger than a tetrahedral angle, but is less than that obtained for the all-trans PDBS $\left(126.50^{\circ}\right)$. This is due to these two angles allow that, in both conformations, $7 / 3$ and all-trans, the smaller distance between the hydrogen atoms is almost equal $(\sim 2.32 \AA)$.

\section{B. Band structure calculations of all-trans poly(di-n- alkylsilanes): Influence of the size of the alkyl group on the electronic properties}

The VEI band structure calculated for all-trans polysilane (PS) ${ }^{29}$ poly(dimethylsilane) (PDMS), and poly (diethylsilane) (PDES) using the helical unit cell $\left(\mathrm{SiR}_{2}\right)$ are displayed in Fig. 2. The band structures calculated for poly(di$n$-propylsilane) (PDPrS) and poly(di- $n$-butylsilane) (PDBS) are very similar to that calculated for PDES and are not displayed in Fig. 2. The electronic bands depicted in this figure are classified as $\sigma$, or $\pi$-like, bands depending on whether one-electron wave functions are either symmetric, or antisymmetric, with respect to the reflection plane containing the silicon backbone.

As can be seen, only four bands are depicted in the band structure of PS, three occupied bands and one virtual. ${ }^{29,30}$ Going from the bottom to the top of the valence band structure, the lowest band is a sigma band mainly built from $3 \mathrm{~s}$ atomic orbitals of silicon atoms but important contributions from hydrogen $1 s$ orbitals and silicon $3 p_{y}$ orbitals are also present ( $x$ axis is the polymeric backbone direction, $y$ axis is that defines the plane that contain the silicon atoms, and $z$ 
axis is the axis perpendicular to this plane). The second occupied band is a $\pi$ band that comes from the bonding interaction of the hydrogen $1 s$ orbitals with the silicon $3 p_{z}$ orbitals. The highest occupied or valence band is a sigma band and is composed of hydrogen $1 s$ and silicon $3 p_{y}$ contributions at the center of the Brillouin zone $(k=0)$, but only silicon $3 p_{x}$ contributions are present at the edges of the Brillouin zone $(k= \pm \pi / a)$, where the highest occupied crystal orbital (HOCO) is located. The lowest unoccupied or conduction band is a sigma band and results from silicon $3 p_{x}$ atomic orbitals at $k=0$, where the lowest unoccupied crystal orbital (LUCO) is located, while silicon $3 s$ and $3 p_{y}$ and hydrogen $1 s$ contributions constitute this band at $k= \pm \pi / a$.

The VEH band structure of PDMS and PDES show similar features to those observed for PS. The main difference is the appearance of couples of $\mathrm{C}-\mathrm{H}$ bands, one symmetric and one antisymmetric respect to the silicon backbone plane, because of the attachment of alkyl groups. The first two bands of PDMS, and the first four bands of PDES, are mainly composed of carbon $2 s$ and hydrogen $1 s$ but weak contributions from silicon orbitals are also observed. These bands are located at the bottom of the diagram, below the first band of PS. The appearance of these bands provokes a shift of the first band of PS to higher energies, which is more pronounced at the bottom of the band. As a consequence, the width of this band decrease in going from PS $(5.04 \mathrm{eV})$ to PDMS $(3.73 \mathrm{eV})$ to PDES $(3.21 \mathrm{eV})$.

Above the first PS band appear the other $\mathrm{C}-\mathrm{H}$ bands, four in PDMS, and eight in PDES. These bands are located approximately between -0.6 and -0.5 atomic units ( 1 a.u. $=27.21 \mathrm{eV}$ ), as can be seen in Fig. 2. They are almost degenerate and show very small bandwidths. In these bands the most important contributions come from hydrogen $1 s$ atomic orbitals. The appearance of these bands produces a shift of the two highest occupied bands of PS to higher energies. The antisymmetric band of both, PDMS and PDES, shows the same shift along the Brillouin zone, and consequently the antisymmetric band of three polymers is parallel and preserves the width. However, the valence band shows a very pronounced shift at the bottom of the band that decreases progressively when moving to the top of the band, and produces a narrowing of the width of the valence band. It should be noted that, as a consequence of this progressively decreasing shift, the edges of the valence band of three polymers, where the HOCO is located, almost coincide. This orbital (HOCO) is mainly composed of silicon $3 p_{x}$ orbitals with weak bonding contributions from $2 p_{x}$ orbitals of the carbons linked to the silicon chain, and from the $1 s$ orbitals of the hydrogen atoms laying out of the $\mathrm{C}_{1} \mathrm{SiC}_{1}^{\prime}$ planes, attached to these carbons.

The attachment of alkyl groups in the polysilane backbone implies the appearance of new unoccupied bands below 0.0 a.u. These bands provoke an important narrowing of the lowest unoccupied band, or conduction band, of PS, that in three polymers (PS, PDMS, and PDES) is a sigma band. However, while in PS the LUCO is located at $k=0$ in both, PDMS and PDES, this orbital is located at $k= \pm \pi / a$. This is due to the bonding interaction between carbon and silicon atoms that takes place at the edges of the Brillouin zone, that
TABLE II. VEH values calculated for Koopmans' ionization potential (IP) and electron affinity (EA), direct band gap $\left(E_{g}\right)$, bandwidth of the highest occupied or valence band (BW-VB), and bandwidth of the lowest unoccupied or conduction band (BW-CB). All the values are in $\mathrm{eV}$.

\begin{tabular}{ccccccc}
\hline Polymer & Conformation & $\mathrm{IP}^{\mathrm{a}}$ & $\mathrm{EA}^{\mathrm{a}}$ & $E_{\mathrm{g}}^{\mathrm{b}}$ & $\mathrm{BW}-\mathrm{VB}$ & $\mathrm{BW}-\mathrm{CB}$ \\
\hline $\mathrm{PS}^{\mathrm{c}}$ & $2 / 1$ & 8.01 & 3.51 & $4.50(k=0)$ & 4.10 & 3.45 \\
PDMS & $2 / 1$ & 7.81 & 4.49 & $3.32(k=0)$ & 3.04 & 1.71 \\
$\mathrm{PDES}$ & $2 / 1$ & 7.74 & 4.29 & $3.45(k=0)$ & 3.01 & 1.20 \\
PDPrS & $2 / 1$ & 7.72 & 4.33 & $3.39(k=0)$ & 3.03 & 1.07 \\
PDBS & $2 / 1$ & 7.78 & 4.42 & $3.37(k=0)$ & 2.95 & 1.04 \\
PDBS & $7 / 3$ & 7.94 & 4.36 & $3.62(k= \pm \pi / a)$ & 2.36 & 1.12 \\
\hline
\end{tabular}

${ }^{a}$ IP and EA values are those obtained for and isolated polymeric chain and no factor has been substracted to take into account the polarization energy of the lattice.

${ }^{b}$ Minimum direct-absorption-type band gaps using translational unit cells. The $k$ point of the Brillouin zone where have been calculated are in parentheses.

${ }^{c}$ Values from Ref. 29.

leads to the stabilization of the crystal orbital at $k= \pm \pi / a$ that becomes the LUCO.

The band structures calculated for larger poly(di- $n$ alkylsilanes), PDPrS and PDBS, are very similar to that obtained for PDES and only an increase of the number of $\mathrm{C}-\mathrm{H}$ bands, with a larger shift of the occupied bands of polysilane to higher energies and a displacement of the unoccupied bands to lower energies is observed.

The VEH values obtained for Koopmans' ionization potentials (IP) and electron affinities (EA), direct band gaps $\left(E_{g}\right)$, and bandwidths of the valence (BW-VB) and conduction bands (BW-CB) of all-trans PS, PDMS, PDES, PDPrS, and PDBS are collected in Table II. As can be seen in the table, the IP values of all organopolysilanes are very similar. This is an expected result since the HOCO is mainly composed of silicon $3 p_{x}$ orbitals, and only small contributions from the alkyl groups are present. The decrease of the IP observed in going from PS $(8.01 \mathrm{eV})$ to poly(di- $n$ alkylsilanes) $(7.7-7.8 \mathrm{eV})$, is mainly due to the increase of the length of the unit cell when passing from PS $(3.86 \AA)$ to poly(di- $n$-alkylsilanes) $(3.92-3.96 \AA)$, as a consequence of the steric hindrance between the alkyl groups. This similarity of the IP is in good agreement with experimental photoemission data obtained for all-trans PDMS (5.73 eV) and alltrans poly(di- $n$-hexylsilane) $(5.78 \mathrm{eV}) .{ }^{44}$ The difference between theoretical and experimental solid-state IP values is typically on the order of $2.0 \mathrm{eV}^{35}$ and is due to polarization effects in solid state. These effects cannot be included, $a$ priori, in band structure calculations, that are performed on isolated chains. The difference between VEH and experimental IP of PDMS is $2.08 \mathrm{eV}$, that is in perfect agreement with the correction factor usually considered to account for the polarization of the solid. ${ }^{35}$

In contrast to the IP, the value calculated for the EA depend on the alkyl group attached to the silicon backbone. The bonding interaction between silicon and carbon atoms that takes place at the edges of the Brillouin zone in PDMS, produces the stabilization of the LUCO of PDMS in relation to that of PS. As a consequence, an important increase of the EA value of PDMS $(4.49 \mathrm{eV})$ with respect to that of PS $(3.58$ 
eV) is observed. When passing to PDES, the increase of the silicon-carbon bond length produces a decrease of the bonding interaction between these atoms, and therefore, the EA value of PDES $(4.29 \mathrm{eV})$ shows a decrease in relation to that of PDMS $(4.49 \mathrm{eV})$. The introduction of larger alkyl groups only leads to a slight progressive increase of the EA values, 4.33 and $4.42 \mathrm{eV}$ for PDPrS and PDBS, respectively, because of the progressive appearance of unoccupied bands below 0.0 a.u, that smoothly move the conduction band, and therefore the LUCO, to lower binding energies.

As can be seen from Table II, the direct band gap $\left(E_{g}\right)$ decreases with the attachment of alkyl groups because of the larger EA values poly(di-n-alkylsilanes) display. The $E_{g}$ value of poly(di-n-alkylsilanes) increases when the size of the alkyl group attached to the silicon backbone decreases (3.37, 3.39, and $3.45 \mathrm{eV}$ for PDBS, PDPrS, and PDES, respectively), except for PDMS (3.32 eV). This behavior is in very good agreement with experimental trends, which show that the energy of the solid-state UV absorption maximum measured for all-trans poly(di-n-alkylsilanes) increases when passing from PDBS $(3.54 \mathrm{cV})^{19,21}$ to PDPrS $(3.60 \mathrm{cV})^{15,19}$ and from this to PDES $(3.73 \mathrm{eV}) .{ }^{15,19}$ In contrast, and unlike that found by other methodologies, ${ }^{24,26}$ when passing from PDES $(3.45 \mathrm{eV})$ to PDMS $(3.32 \mathrm{eV})$ a decrease of the $E_{g}$ value is observed. This is due to the larger EA value PDMS presents, as a consequence of the short $\mathrm{Si}-\mathrm{C}$ bond length. This different behavior coincides with that observed experimentally for $\lambda_{\max }$ values of these polymers [3.73 and $3.63 \mathrm{eV}$ for PDES ${ }^{15,19}$ and PDMS, ${ }^{16,45}$ respectively].

\section{Influence of the backbone conformation on the electronic properties of PDBS}

Among the polymers studied in this paper only PDBS has shown experimental evidences of both, all-trans and $7 / 3$ conformations. The $7 / 3$ helical conformation is the most stable conformation at atmospheric pressure, ${ }^{17}$ and only when the pressure is increased the all-trans conformation is observed..$^{21}$ In this section we compare the electronic properties of PDBS in both, all-trans and 7/3 conformations.

The VEH electronic band structure calculated for $7 / 3$ PDBS is totally similar to that calculated for all-trans PDBS, and only small differences, in the width of the bands, are appreciated. The VEH values obtained for the electronic properties of 7/3 PDBS have been collected in Table II, together with those of all-trans poly(di- $n$-alkylsilanes). In going from all-trans to $7 / 3$ conformation a decrease of the interactions between the silicon $3 p_{x}$ atomic orbitals ( $x$ axis corresponds to the polymeric backbone direction) that constitutes the $\mathrm{HOCO}$ is observed. This is due to the minor overlap of the $3 p_{x}$ orbitals in $7 / 3$ conformation. This decrease induces a narrowing of the valence band $(2.95 \rightarrow 2.36$ $\mathrm{eV})$ and therefore, a reduction of the delocalization of the electrons in this band. This behavior is similar to that observed previously when passing from all-trans to $7 / 3 \mathrm{PS},{ }^{30}$ where the width of the valence band decreased from 4.10 to $3.50 \mathrm{eV}$. This narrowing leads to an increase of the value of the ionization potential, which goes from $7.78 \mathrm{eV}$ (all-trans) to $7.94 \mathrm{eV}(7 / 3)$. This increase is in perfect agreemenl with that observed experimentally for the ionization potential of PDHS, when passing from the ordered all-trans conformation $(5.78 \mathrm{eV})$, to the disordered phase $(5.94 \mathrm{eV}),{ }^{44}$ in which a melting of gauche and all-trans sequences is present.

The LUCO of all-trans PDBS is mainly composed of $3 s$ and $3 p_{y}$ atomic orbitals of the silicon atoms, and $2 s$ and $2 p_{z}$ orbitals of the carbon atom linked to the silicon atom, with weak contributions from the other carbons forming the butyl group. When passing to the $7 / 3$ conformation the antibonding interaction between the $3 s$ atomic orbitals of the silicon atoms increase, because of the major proximity of the silicon atoms (see Table I). Therefore, the LUCO is slightly destabilized and produces a change of $0.06 \mathrm{eV}$ in the $\mathrm{EA}$ value, which decreases from $4.42 \mathrm{eV}$ in the all-trans to $4.36 \mathrm{eV}$ in the $7 / 3$ conformation.

As a consequence of the increase of the IP and the decrease of the EA values the band gap of PDBS increases 0.25 $\mathrm{eV}$ when passing from the all-trans $(3.37 \mathrm{eV})$ to the $7 / 3(3.62$ eV) conformation, that correlates with VEH calculations on PS $(0.18 \mathrm{eV})^{30}$ and EHT calculations on PDMS $(0.3 \mathrm{eV}){ }^{27}$ This increase is in very good agrecment with the red-shift of $0.40 \mathrm{eV}$ observed experimentally for the UV absorption maximum of PDBS films in going from a $7 / 3(315 \mathrm{~nm}, 3.94$ $\mathrm{eV})^{17}$ to an all-trans $(350 \mathrm{~nm}, 3.54 \mathrm{eV})^{21}$ conformation, when the pressure is increased.

\section{SUMMARY AND CONCLUSIONS}

We have investigated the evolution of the geometric and electronic structure of poly(di-n-alkylsilanes). On one hand we have analyzed polysilane, poly(dimethylsilane), poly(diethylsilane), poly(di- $n$-propylsilane), and poly(di- $n$ butylsilane) in the all-trans conformation. We have studied in detail the changes induced in the geometry and VEH band structure by the increase of the size of the alkyl group. On the other hand, we have compared poly(di- $n$-butylsilane) in the all-trans and $7 / 3$ conformations, in order to analyze the influence of the backbone conformation on the geometrical and electronic properties.

The geometric parameters, bond lengths, and bond angles, obtained at the $3-21 \mathrm{G}^{*}$ show that methylation only induces small changes in the $\mathrm{Si}-\mathrm{Si}$ bond length $(2.3456 \rightarrow 2.3522 \AA)$ which are more accentuated when passing to the $\angle$ SiSiSi bond angle $\left(110.91 \rightarrow 112.93^{\circ}\right)$ because of the steric hindrance between methyl groups in 1 and 3 positions. The change of methyl groups by ethyl groups, when passing from PDMS to PDES, produces an increase of both, $\mathrm{Si}-\mathrm{Si}(0.035 \AA)$ and $\mathrm{Si}-\mathrm{C}_{1}(0.027 \AA)$, bond length. The $\angle C_{1}$ SiSi bond angle increases from $108.72^{\circ}$ to $112.79^{\circ}$ and consequently the $\angle \mathrm{SiSiSi}$ bond angle decreases from $112.93^{\circ}$ to $111.57^{\circ}$. The $\angle \mathrm{C}_{2} \mathrm{C}_{1} \mathrm{Si}$ bond angle displays an important deviation from a typical tetrahedral angle, because of the strong interaction between the hydrogens of the terminal carbon of the ethyl group that are linked to consecutive silicon atoms. The geometrical parameters calculated for alltrans PDPrS and PDBS are totally similar to that obtained for all-trans PDES. The geometry of both, all-trans and $7 / 3$ PDBS are very similar except for the $\mathrm{Si}-\mathrm{Si}$ bond length that shows and important decrease $(2.3876 \AA$ for all-trans and 
$2.3594 \AA$ for $7 / 3$ ) and the $\angle \mathrm{C}_{1} \mathrm{SiSi}$ and $\angle \mathrm{C}_{2} \mathrm{C}_{1} \mathrm{Si}$ bond angles that show a decrease of about $6.1^{\circ}$, in order to decrease the interactions between alkyl groups.

The VEH electronic structure calculated for poly(di- $n$ alkylsilanes) shows that the increase of the size of the alkyl group produces the appearance of new couples of $\mathrm{C}-\mathrm{H}$ bands. These bands produce the shift of the occupied bands of PS to higher energies, a displacement of the unoccupied bands to lower energies and a narrowing of the width of these bands.

The IP displays similar values in going from PDMS to PDBS (7.7-7.8 eV), which is in good agreement with experimental photoemission data obtained for all-trans PDMS $(5.73 \mathrm{eV})$ and all-trans poly (di-n-hexylsilane) $(5.78 \mathrm{eV})$. The direct band gap decreases when the size of the alkyl group increases $(3.45,3.39$, and $3.37 \mathrm{eV}$ for PDES, PDPrS, and PDBS, respectively) except for PDMS (3.32 eV). These features are in good agreement with the shift measured experimentally for the energy of the solid-state UV absorption maximum of all-trans poly(di- $n$-alkylsilanes).

Both IP and $E_{g}$ of PDBS increase when passing from all-trans to $7 / 3$ conformation. The IP goes from $7.78 \mathrm{eV}$ (all-trans) to $7.94 \mathrm{eV}(7 / 3)$, which coincides with the difference of $0.16 \mathrm{eV}$ observed experimentally for PDHS, when passing from an all-trans to a disordered phase. The increase of the $E_{g}$ of PDBS in going from all-trans $(3.37 \mathrm{eV}$ ) to $7 / 3$ $(3.62 \mathrm{eV})$ conformation is in very good agreement with the red-shift observed for the UV absorption maximum of PDBS films $(0.4 \mathrm{eV})$.

The very good agreement between VEH and experimental values for the IP and $E_{g}$ when increasing the size of the alkyl group, and when change the backbone conformation, shows the applicability of the VEH technique to deal with organopolysilanes.

\section{ACKNOWLEDGMENTS}

This research was supported by the Generalitat Valenciana and the DGICYT Projects PB91-0935 and OP90-0042. M.C.P. is grateful to the Ministerio de Educación y Ciencia of Spain for a Doctoral Grant. We thank Wladimiro Diaz and the Servei d'Informàtica de la Universitat de València for helpful technical support.

'In Proceedings of the ICSM'92 (International Conference on Science and Technology of Synthetic Metals), Synth. Met., Vol. 55-57, (1993).

${ }^{2}$ R. D. Miller and J. Michl, Chem. Rev. 89, 1359 (1989).

${ }^{3}$ Silicon-Based Polymer Science. A Comprehensive Resource, Advances in Chemistry Series, edited by J. M. Zeigler and F. G. Fearon (American Chemical Society, Washington, D.C., 1990), Vol. 224; J. E. Mark, H. R. Allcock, and R. West, Inorganic Polymers (Prentice-Hall, Englewood Cliffs, 1992), Chap. 5, p. 186.

${ }^{4}$ S. Gauthier and D. J. Worsfold, Macromolecules 22, 2213 (1989); R. D. Miller, D. Hofer, G. N. Fickes, C. G. Willson, E. Marinero, P. Trefonas III, and R. West, Polym. Eng. Sci. 26, 1129 (1986).

${ }^{5}$ R. G. Kepler, J. M. Zeigler, L. A. Harrah, and S. R. Kurtz, Phys. Rev. B 35, 2818 (1987); M. Stolka, H.-J. Yuh, K. McGrane, and D. M. Pai, J. Polym. Sci. Polym. Chem. Ed. 25, 823 (1987).

${ }^{6}$ R. West, A. R. Wolff, and D. J. Peterson, J. Radiat. Curing 13, 35 (1986); A. R. Wolff and R. West, Appl. Organomet. Chem. 1, 7 (1987).

'K. Kajzar, J. Messier, and C. Rosilio, J. Appl. Phys. 60, 3040 (1986); P. Shukla, P. M. Cotts, R. D. Miller, S. Ducharme, R. Asthana, and J. ZavisIan, Mol. Cryst. Liquid Cryst. 183, 241 (1990).
${ }^{8}$ P. 'Trefonas IIl, R. West, R. D. Miller, and D. Hofer, J. Polym. Sci. Polym. Lett. Ed. 21, 823 (1983).

${ }^{9}$ L. A. Harrah and J. M. Zeigler, Macromolecules 20, 601 (1987).

${ }^{10}$ L. A. Harrah and J. M. Zeigler, in Photophysics of Polymers, ACS Symposium Series, edited by C. E. Hoyle and J. M. Torkelson (American Chemical Society, Washington, D.C., 1987), Vol. 358, p. 482.

${ }^{11}$ L. A. Harrah and J. M. Zeigler, J. Polym. Sci. Polym. Lett. Ed. 23, 209 (1985); P. Trefonas III, J. R. Damewood, Jr., R. West, and R. D. Miller, Organometall. 4, 1318 (1985).

${ }^{12}$ R. D. Miller, D. Hofer, J. Rabolt, and G. N. Fickes, J. Am. Chem. Soc. 107, 2172 (1985).

${ }^{13}$ A. J. Lovinger, F. C. Schilling, F. A. Bovey, and J. M. Zeigler, Macromolecules 19, 2657 (1986); F. C. Schilling, F. A. Bovey, A. J. Lovinger, and J. M. Zeigler, ibid. 19, 2660 (1986); H. Kuzmany, J. F. Rabolt, B. L. Farmer, and R. D. Miller, J. Chem. Phys. 85, 7413 (1986).

${ }^{14}$ J. F. Rabolt, D. Hofer, R. D. Miller, and G. N. Fickes, Macromolecules 19, 611 (1986).

${ }^{15}$ A. J. Lovinger, D. D. Davis, F. C. Schilling, F. A. Bovey, and J. M. Zeigler, Polym. Commun. 30, 356 (1989).

${ }^{16}$ A. J. Lovinger, D. D. Davis, F. C. Schilling, F. J. Padden, Jr., F. A. Bovey, and J. M. Zeigler, Macromolecules 24, 132 (1991).

${ }^{17}$ F. C. Schilling, A. J. Lovinger, J. M. Zeigler, D. D. Davis, and F. A. Bovey, Macromolecules 22, 3055 (1989).

${ }^{18}$ R. D. Miller, B. L. Farmer, W. Fleming, R. Sooriyakumaran, and J. F. Rabolt, J. Am. Chem. Soc. 109, 2509 (1987).

${ }^{19}$ F. C. Schilling, F. A. Bovey, D. D. Davis, A. J. Lovinger, R. B. MacGregor, Jr., C. A. Walsh, and J. M. Zeigler, Macromolecules 22, 4645 (1989).

${ }^{20}$ K. Song, H. Kuzmany, G. M. Wallraff, R. D. Miller, and J. F. Rabolt, Macromolecules 23, 3870 (1990).

${ }^{21}$ K. Song, R. D. Miller, G. M. Wallraff, and J. F. Rabolt, Macromolecules 24, 4084 (1991).

${ }^{22}$ R. W. Bigelow, Organometall. 5, 1502 (1986); Chem. Phys. Lett. 126, 63 (1986).

${ }^{23}$ K. A. Klingensmith, J. W. Downing, R. D. Miller, and J. Michl, J. Am. Chem. Soc. 108, 7438 (1986).

${ }^{24} \mathrm{~K}$. Takeda, H. Teramae, and N. Matsumoto, J. Am. Chem. Soc. 108, 8186 (1986); K. Takeda, M. Fujino, K. Seki, and H. Inokuchi, Phys. Rev. B 36, 8129 (1987); N. Matsumoto, K. Takeda, H. Teramae, and M. Fujino, in Silicon-Based Polymer Science. A Comprehensive Resource, Advances in Chemistry Series, edited by J. M. Zeigler and F. G. Fearon (American Chemical Society, Washington, D.C., 1990), Vol, 224, p. 515.

${ }^{25}$ H. Teramae and K. Takeda, J. Am. Chem. Soc. 111, 1281 (1989); N. Matsumoto and H. Teramae, ibid. 113, 4481 (1991); J. M. André, Int. J. Quantum Chem. 24, 65 (1990).

${ }^{26}$ J. W. Mintmire, Phys. Rev. B 39, 13350 (1989); J. W. Mintmire and J. V. Ortiz, Polym. Prep. 31, 234 (1990); in Silicon-Based Polymer Science. A Comprehensive Resource, Advances in Chemistry Series, edited by $\mathbf{J}$. M. Zeigler and F. G. Fearon (American Chemical Society, Washington, D.C., 1990), Vol. 224, p. 543.

${ }^{27}$ C. X. Cui, A. Karpfen, and M. Kertesz, Macromolecules 23, 3302 (1990).

${ }^{28}$ R. Crespo, M. C. Piqueras, E. Ortí, and J. L. Brédas, Synth. Met. 43, 3457 (1991).

${ }^{29}$ R. Crespo, M. C. Piqueras, E. Ortí, and F. Tomás, Synth. Met. 54, 173 (1993).

${ }^{30}$ E. Orti, R. Crespo, M. C. Piqueras, F. Tomás, and J. L. Brédas, Synth. Met. 57, 4419 (1993).

${ }^{3 \mathrm{I}}$ R. Crespo, M. C. Piqueras, E. Ortí, and F. Tomás, Synth. Met. 61, 107 (1993).

${ }^{32}$ Gaussian 92, M. J. Frisch, G. W. Trucks, M. Head-Gordon, P. M. W. Gill, M. W. Wong, J. B. Foresman, B. G. Johnson, H. B. Schlegel, M. A. Robb, E. S. Replogle, R. Gomperts, J. L. Andres, K. Raghavachari, J. S. Binkley, C. Gonzalez, R. L. Martin, D. J. Fox, D. J. Defrees, J. Baker, J. J. P. Stewart, and J. A. Pople, Gaussian, Inc., Pittsburgh, Pennsylvania, 1992.

${ }^{33} \mathrm{~J}$. M. André, J. L. Brédas, J. Delhalle, D. J. Vanderveken, D. P. Vercauteren, and J. G. Fripiat, in Modern Techniques in Computational Chemistry: MOTECC-90, edited by E. Clementi (Escom, Leiden, 1990), Chap. 15, p. 745

${ }^{34}$ J. M. André, J. Delhalle, and J. L. Brédas, Quantum Chemistry Aided Design of Organic Polymers. An Introduction to the Quantum Chemistry of Polymers and its Aplications (World Scientific, Singapore, 1991), Chap. 3, p. 163.

${ }^{35}$ J. L. Brédas, in Handbook of Conducting Polymers, edited by T. A. Skotheim (Dekker, New York, 1986), Vol. 2, Chap. 25, p. 859. 
${ }^{36}$ G. Nicolas and Ph. Durand, J. Chem. Phys. 70, 2020 (1979); 72, 453 (1980).

${ }^{37}$ J. M. André, L. A. Burke, J. Delhalle, G. Nicolas, and Ph. Durand, Int. J. Quantum Chem. Symp. 13, 283 (1979).

${ }^{38}$ E. Ortí, M. C. Piqueras, R. Crespo, and J. L. Brédas, Chem. Mater, 2, 110 (1990); E. Ortfai, R. Crespo, M. C. Piqueras, and J. L. Brédas, Synth. Met. 42, 2647 (1991); E. Orti, M. C. Piqueras, R. Crespo, and F. Tomás, ibid. 57, 4519 (1993).

${ }^{39}$ J. Delhalle, L. Piela, J. L. Brédas, and J. M. André, Phys. Rev. B 22, 6254 (1980), and references therein.

${ }^{40}$ J. L. Brédas, R. R. Chance, R. Silbey, G. Nicolas, and Ph. Durand, J. Chem. Phys. 75, 255 (1981).
${ }^{41}$ J. M. André, J. Delhalle, and J. L. Brédas, Quantum Chemistry Aided Design of Organic Polymers. An Introduction to the Quantum Chemistry of Polymers and its Aplications (World Scientific, Singapore, 1991), Chap. 2, p. 77.

${ }^{42}$ J. T. Nelson and W. J. Pietro, J. Phys. Chem. 92, 1365 (1988); J. V. Ortiz and J. W. Mintmire, J. Am. Chem. Soc. 110, 4522 (1988).

${ }^{43}$ W. J. Pietro, M. M. Francl, W. J. Hehre, D. J. Defrees, J. A. Pople, and J. S. Binkley, J. Am. Chem. Soc. 104, 5039 (1982).

${ }^{44}$ K. Yokoyama and M. Yokoyama, Solid State Commun. 70, 241 (1989).

${ }^{45}$ L. A. Leites, S. S. Bukalov, T. S. Yadritzeva, M. K. Mokhov, B. A. Antipova, T. M. Frunze, and V. V. Dement'ev, Macromolecules 25, 2991 (1992). 